\title{
Early Childhood Exposure to Anesthesia and Risk of Developmental and Behavioral Disorders in a Sibling Birth Cohort
}

\author{
Charles DiMaggio, PhD, \\ Department of Anesthesiology, Columbia University, New York, NY \\ Lena Sun, MD, and \\ Department of Anesthesiology, Columbia University, New York, NY \\ Guohua Li, MD, DrPH \\ Department of Anesthesiology, Columbia University, New York, NY
}

\begin{abstract}
Introduction-In vitro and in vivo studies of anesthetics have demonstrated serious neurotoxic effects on the developing brain. However the clinical relevance of these findings to children undergoing anesthesia remains unclear. Using data from a sibling birth cohort, we assessed the association between exposure to anesthesia in the setting of surgery in patients younger than 3 years and the risk of developmental and behavioral disorders.

Methods-We constructed a retrospective cohort of 10,450 siblings who were born between 1999 and 2005 and who were enrolled in the New York State Medicaid program. The exposed group was 304 children without a history of developmental or behavioral disorders who underwent surgery when they were younger than 3 years. The unexposed group was 10,146 children who did
\end{abstract}

Corresponding Author: Charles DiMaggio, PhD Columbia University 622 West 168 Street New York, NY 10032 Phone: 212.305.0019 FAX: 212.305.3296 cjd11@columbia.edu.

The authors declare no conflicts of interest.

Reprints will not be available from the authors.

DISCLOSURES All the authors made substantive contributions to the conceptualization and design of the study and all endorse the results and conclusions.

Name: Charles DiMaggio, PhD

Contribution: This author helped design the study, conducted the study, analyzed the data, wrote the first draft, and helped obtain grant funding. Dr. DiMaggio, through a data use agreement with the US Centers for Medicare and Medicaid Services, had full access to all the data in the study and takes responsibility for the integrity of the data and the accuracy of the analyses. He was also responsible for obtaining IRB approval, the initial drafting of the manuscript, as well as for critical revisions.

Attestation: Charles DiMaggio has seen the original study data, reviewed the analysis of the data, approved the final manuscript, and is the author responsible for archiving the study files.

Name: Lena Sun, MD

Contribution: This author helped design the study, conduct the study, and write the manuscript. Dr. Sun suggested and guided analyses, and contributed to the interpretation of the data, drafting of the manuscript, and critical revision of the manuscript for important intellectual content.

Attestation: Lena Sun reviewed the analysis of the data and approved the final manuscript.

Name: Guohua Li, MD, DrPH

Contribution: Dr. Li contributed to the study design, statistical analysis, interpretation of the results, drafting of the manuscript, and critical revision of the manuscript for important intellectual content.

Attestation: Guohua Li has seen the original study data, reviewed the analysis of the data, and approved the final manuscript.

This is a PDF file of an unedited manuscript that has been accepted for publication. As a service to our customers we are providing this early version of the manuscript. The manuscript will undergo copyediting, typesetting, and review of the resulting proof before it is published in its final citable form. Please note that during the production process errors may be discovered which could affect the content, and all legal disclaimers that apply to the journal pertain. 
not receive any surgical procedures when they were younger than 3 years. Exposed children were entered into analysis at the date of surgery. Unexposed children were entered into analysis at age 10 months (the mean age at which exposed children underwent surgery). Both exposed and unexposed children were followed until diagnosis with a developmental or behavioral disorder, loss to follow-up, or the end of 2005. The association of exposure to anesthesia with subsequent developmental and behavioral disorders was assessed with both proportional hazards modeling, and pair-matched analysis.

Results-The incidence of developmental and behavioral disorders was 128.2 diagnoses per 1000 person-years for the exposed cohort and 56.3 diagnoses per 1000 person-years for the unexposed cohort. With adjustment for sex and history of birth-related medical complications, and clustering by sibling status, the estimated hazard ratio of developmental or behavioral disorders associated with any exposure to anesthesia when they were younger than 3 years was 1.6 (95\% CI $1.4,1.8)$. The risk increased from $1.1(95 \% \mathrm{CI} 0.8,1.4)$ for one surgery to $2.9(94 \% \mathrm{CI} 2.5,3.1)$ for two surgeries and 4.0 (95\% CI 3.5, 4.5) for three or more surgeries. The risk in a matched analysis of 138 sibling pairs was $0.9(95 \%$ CI $0.6,1.4)$

Conclusion-The risk of being subsequently diagnosed with developmental and behavioral disorders in children who were enrolled in a state Medicaid program and who had surgery when they were younger than 3 years was $60 \%$ greater than that of a similar group of siblings who did not undergo surgery. More tightly matched pairwise analyses indicate that the extent to which the excess risk is causally attributable to anesthesia or mediated by unmeasured factors remains to be determined.

\section{INTRODUCTION}

Laboratory studies have demonstrated that commonly used anesthetics have neurotoxic effects in infant rats and other animals. ${ }^{1,2}$ These effects are thought to be mediated through actions at the N-methyl-D-aspartic acid glutamate receptors and/or $\gamma$-aminobutyric acid receptors, ${ }^{3}$ a process similarly proposed for fetal alcohol syndrome. 4

Since first described, the laboratory evidence has accumulated with repeated demonstrations of anesthesia-induced neuronal damage and death in vitro and impaired neurobehavioral functions in vivo under varying experimental conditions, and investigations of potential interventions to mitigate against their effects. ${ }^{5-7}$

The clinical relevance of these studies has been called into doubt. Infant rats have a relatively short vulnerable synaptogenic period in contrast to humans, and relatively high doses of anesthetics and long duration of anesthetic exposure have been used to trigger apoptosis, indicating that any extrapolation to humans would require frequent, repeated exposure over a long period of time. The effects in rodents might be due to hypoxia, rather than anesthetic agents. This is prevented in the clinical setting through careful and continuous monitoring. ${ }^{8}$ Notably, the studies were not performed in animals undergoing surgery, and in one instance in which animals received anesthesia in the presence of inflammation, simulating surgical anesthesia, ketamine attenuated the impaired neurocognitive behaviors induced by neonatal exposure to inflammatory pain. ${ }^{9}$

Ultimately, the experimental studies cannot include those variables that are important in the clinical setting such as maternal history and the use of antecedent treatment during pregnancy, labor and delivery, confounding factors due to indications for surgery and preexisting conditions, and environmental or ecological characteristics. 
At least two prospective studies are underway to determine whether the experimental animal-based evidence has implications for humans. ${ }^{10,11}$ In the meantime, the clinical evidence to date consists of analyses of data with sometimes-conflicting results.

A population-based retrospective cohort of anesthesia exposure in the 1970's and 1980s found a $60 \%$ increased risk after more than one anesthetic exposure. ${ }^{12}$. A retrospective cohort analysis of 1991-2002 New York State Medicaid data found a two-fold increased risk for having had inguinal surgery before age 3 years and subsequent developmental or behavioral diagnoses. ${ }^{13}$ Both studies controlled, as much as the data would allow, for preexisting medical conditions, age, gender and socioeconomic status. More recently, a study based on analyses of a Dutch twin registry did not find conclusive evidence for a causal relationship between exposure to anesthesia and subsequent cognitive deficit. ${ }^{14}$

Our purpose in this study was to assess the association of anesthesia in the setting of surgery with subsequent developmental or behavioral diagnoses in a retrospective birth cohort of siblings nested in a previously studied cohort of children from whom an association between anesthesia and developmental outcomes has been demonstrated. ${ }^{13}$ By establishing sibling status and comparing exposed to unexposed siblings, we aimed to control for important variables such as age, home environment, parenting style, educational systems and neighborhood or ecological characteristics.

\section{MATERIALS AND METHODS}

The study protocol was reviewed and approved as exempt by the Columbia University IRB.

\section{Data Sources}

The study was based on New York State Medicaid Analytic Extract (MAX) files from 1999 to $2005 .{ }^{15}$ Medicaid MAX files are a complete set of person-level data files on all state residents who received Medicaid-funded inpatient, outpatient, and long-term care service use including prescription drug use.

Medicaid is a federal, state, and, in New York State, a locally funded health insurance program with locally determined income, age, and disability requirements. To be eligible for Medicaid, a person must belong to a "Category" and meet strict financial tests. The general eligibility categories are (1) poor children and their parents (2) the disabled and (3) the elderly. Medicaid provides coverage to $26 \%$ of all children in the United States (50\% of all low-income children), $37 \%$ of all pregnant women, and $20 \%$ of all adults. ${ }^{16}$ New York State is among the more generous Medicaid programs and covers approximately $50 \%$ of all births in the state. ${ }^{17}$

This administratively collected large dataset consists of 4 separate files: demographic characteristics of enrollees, inpatient admission and discharge data, outpatient service use including clinic, private physician and emergency department care and prescription medication records. The files are linkable through a Medicaid identification number. Available variables include: patient age, gender, zip code of residence, ethnicity, language spoken, beginning and ending dates of eligibility, dates of service, length of stay, principal and up to 9 additional International Classification of Diseases, Ninth Revision-Clinical Modification (ICD-9-CM) diagnosis codes, procedure dates and codes, place of service (e.g. emergency department, outpatient clinic), provider identification codes, prescription dates and national drug codes for medications as well as cost of care. ${ }^{18}$

The data files are compiled by the Centers for Medicare and Medicaid Services (CMS) from claim information provided by the New York State Department of Health. Health care 
providers submit standardized claims ${ }^{19}$ that include clinical information that is commonly abstracted from patient charts by nonclinician administrative personnel. Demographic data such as race and ethnicity designations are based on administrative data collected by CMS from information provided by enrollees as part of their application. CMS routinely conducts validation studies of MAX data and researchers have analyzed and evaluated the reliability of these data for studies of outpatient psychiatric and behavioral diagnoses in New York State. ${ }^{20}$

\section{Birth Cohort}

We searched Medicaid MAX files for system-specific codes for maternal and newborn admissions as well as delivery-related first or primary ICD-9 "V series" diagnosis codes to identify children who were born in New York state between 1999 and 2005. For each uniquely identified birth, we searched primary and secondary ICD-9 codes for any of 41 delivery or birth-related diagnosis that would indicate a complication at the time of birth.*

We identified 635,758 complete New York State Medicaid entries for births between 1999 and 2005 . There was a $17.3 \%$ loss to follow-up over four years, with no statistically significant difference in the rate of surgical procedures between children lost to follow-up and those who remained in the cohort. ${ }^{13}$ Children who were lost to follow-up were censored from analysis on the final calendar date of the final year for which they were enrolled in Medicaid.

After excluding entries for routine interventions such as vaccinations, antibiotic administration, and physical examination, there were 243,238 procedures among the 635,758 births. Circumcision, which accounted for 164,672 (67.7\%) of the 243,238 procedures, was also excluded. Approximately $9 \%(60,319)$ of the children born into New York State Medicaid between 1999 and 2005 received a developmental or behavioral diagnosis by the end of 2005, the most common (73.3\%) being "developmental delay."

\section{Sibling Cohort}

From the 635,758 individual births, we identified 15,781 births in the New York State Medicaid population with ICD-9 codes for twin-sibling deliveries ("V3300," "V330," "V3301," "V310," "V3100," "V3101"). These 15,781 births were exclusive of multiple births with more than two babies from a single pregnancy. Using a combination of the variables for date of birth, zip code of residence, provider identifier, and hospital identifier we successfully matched 11,648 twin-sibling births (73.8\%) into 5,824 twin-sibling pairs. We were unable to match the remaining births due to missing or miscoded variables. The unmatched children were, on average, 5 months older than the children we were able to successfully match ( 44 months vs. 39 months, $\mathrm{p}=0.001$ ). They did not differ from children who we were able to successfully match in terms of gender, surgical/anesthetic exposure, or behavioral/developmental diagnoses.

\section{Exposures}

We defined exposure as an inpatient or outpatient ICD9 procedure code indicative of exposure to general anesthesia in a child younger than 3 years who had no previous developmental or behavioral diagnosis. Exposure status was determined using procedure codes because there is no explicit code for anesthesia exposure, and because it is not possible to consider anesthesia separate from the surgical procedure for which it is administered.

\footnotetext{
${ }^{*}$ A list of the ICD-9 codes used to identify birth-related complications is included as an Appendix.
} 
The definition of a billable procedure code is set by New York State Medicaid and applied consistently across all hospitals. We treated multiple procedure codes for the same date of service as a single exposure with the type of procedure defined by the primary billable procedure.

There were 668 procedure codes before age 3 years for 11,648 children. We excluded 364 children who had a preexisting developmental or behavioral diagnosis before undergoing a surgical procedure. We also excluded 23 neurosurgical, 25 cardiac, 9 palatal, and 2 diaphragmatic procedures. This restriction was based on an assumption that these children had preexisting conditions likely to result in the types of behavioral/developmental diagnoses in which we were interested and that these procedures would confound the relationship between surgery/anesthesia and behavioral/developmental disorders.

The exposed group consisted of 304 children with a record of a surgical/anesthetic exposure before age three years and no recorded history of a developmental or behavioral diagnosis before the date of surgery. The unexposed group consisted of 10,146 children with no recorded history of surgery/anesthesia before age 3 years and no recorded history of a developmental or behavioral diagnosis before age 10 months (the average age at which exposed children were diagnosed). Children in the unexposed cohort who were diagnosed with a developmental or behavioral disorder before age 10 months were excluded from the analysis. Consequently, there were 304 children in the exposed cohort and 10,146 children in the unexposed cohort. A flow chart detailing the selection of study subjects is presented in figure 1.

\section{Outcomes}

We defined our outcome as an ICD9 diagnosis code for autism ('200' and '20081'), unsocial and social conduct disorders ('312,' '31212,' '31220' and '31223'), developmental delay ('3154' and '3159'), reading and language disorders ('3149' and '31539'), attention deficit and hyperkinetic disorders ('31400' and ' 3149 '), and other emotional or conduct disorders ('31239' and '3139'). We chose this group of diagnoses because there is no current best evidence on what, if any cognitive, neurodevelopmental or behavioral outcomes may be associated with anesthesia and these disorders represent a fairly broad spectrum of potential behavioral/developmental problems in early childhood.

\section{Analysis}

We tabulated information on patient age in months, gender, race, diagnoses of complications at birth, exposure procedures and outcome diagnoses, dates of exposure, whether exposure procedures were conducted as an inpatient or an outpatient, dates of outcome diagnoses, and dates of loss to follow-up.

For exposed children, person-years of follow-up were calculated from the date of surgical procedure until the date of a behavioral/developmental diagnosis, loss to follow-up or censoring due to the end of the study on 31 December 2005. For unexposed children, person-years of follow-up were calculated from age 10 months (the average age at which exposed children underwent a surgical procedure) until the date of a behavioral/ developmental diagnosis or censoring due to the end of the study on 31 December 2005 or loss to follow-up.

We determined surgery/anesthesia exposure rates, incidence of behavioral and developmental diagnoses among exposed and unexposed children and calculated rate ratios and Kaplan-Meier survival curves for censoring up to either date of outcome diagnosis or end of study. We conducted proportional hazards modeling controlling for confounding due to medical history of birth-related complications such as low-birth weight, and for male 
gender. We controlled for matching due to sibling status by including a clustering variable. ${ }^{21} \mathrm{We}$ conducted an additional pair-matched analyses conditioned on sibling status and gender calculating an adjusted relative risk estimate. ${ }^{22-24}$

As a sensitivity analysis we assessed the potential magnitude of unmeasured confounding by repeating our calculations for the association of exposure to surgery/anesthesia with behavioral/developmental diagnosis restricted to children who received an outcome diagnosis before being exposed. Follow-up for this group was calculated from date of birth until the date of surgery. The unexposed group for this comparison consisted of children who did not have surgery when they were younger than 3 years followed until the average age of surgery in the exposed group.

Datasets were created using PROC SQL 25 and analyzed using both SAS 9.1 and the R statistical program. ${ }^{26}$

\section{RESULTS}

\section{Unmatched Analysis of The Study Group}

Of the 304 exposed children, 210 (68\%) had a single exposure, 71 (23\%) had two exposures, $23(8 \%)$ had 3 or more exposures. More than half (54\%) of surgical procedures involved an inpatient admission. The most frequent surgical exposure was inguinal hernia repair. (Table 1) The average age at which an exposed child underwent a procedure was 10 months (95\% CI 7 months, 14 months).

Among these 304 children who had a surgical / anesthetic exposure as defined for the study, 75 subsequently received a developmental or behavioral diagnosis. The observation period for exposed children from time of procedure to time of diagnosis, study end or loss to follow-up was 585 person years, for an incidence rate of 128.2 developmental or behavioral diagnoses per 1,000 person-years of observation (75 diagnoses / 585 person-years $\times 1000$ ). The average age at which an exposed child received a developmental or behavioral diagnosis was 18 months (95\% CI 14, 22 months).

Of the 10,146 unexposed children, 896 received a developmental or behavioral diagnosis after 10 months of age. The total observation period for unexposed children, calculated from age 10 months to day of diagnosis, loss to follow-up or study end, totaled 15,917 person years. The incidence rate for outcomes among unexposed children was 56.3 developmental or behavioral diagnoses per 1,000 person-years of observation (896 diagnoses / 15,917 person-years $\times 1000$ ). The most common developmental or behavioral diagnosis for both exposed and unexposed children was "unspecified developmental delay." (Table 2)

One hundred ninety-eight $(65.4 \%)$ of the 304 exposed children had a history of a complication at birth, the most common of which were low birth weight $(93 / 304=30.7 \%)$, followed by perinatal infection $(60 / 304=19.8 \%)$ and perinatal hypoxia $(31 / 304=10.2 \%)$. Among the unexposed children 6,542 (59.9\%) had a history of a complication at birth, with $3,052(27.8 \%)$ having a diagnosis of low birth weight, $2,384(21.6 \%)$ having a diagnosis of perinatal infection and $610(5.5 \%)$ having a diagnosis of perinatal hypoxia.

In a proportional hazards model controlling for a history of complication at birth and gender and including a clustering variable for sibling status, the hazard ratio for the association of any prior surgery/anesthesia with subsequent behavioral/developmental diagnosis was 1.6 $(95 \%$ CI 1.4, 2.8). In this model, the hazard ratio was $1.4(95 \%$ CI $1.3,1.5)$ for male gender and $1.5(95 \%$ CI $1.4,1.6)$ for a history of complication at birth. The Kaplan-Meir survival curve for any exposure adjusted for birth complications and gender is presented in Figure 2. 
When the analysis was restricted to only children who did not have a history of a birth complication, the hazard ratio associated with prior surgery/anesthesia was 1.6 (95\% CI 1.1, $2.0)$ and that for gender was 1.7 (95\% CI 1.4, 1.9).

Ninety-four of the 304 exposed children had more than one surgical procedure during the study period. In a proportional hazards model assessing the association of multiple surgical/ anesthetic exposures with subsequent behavioral/developmental diagnosis the hazard ratio was $1.1(95 \%$ CI $0.8,1.4)$ for a single exposure, 2.8 (95\% CI $2.5,3.1)$ for two exposures, and $4.0(95 \%$ CI $3.5,4.4)$ for three or more exposures (Table 3$)$

\section{Sibling-Matched Analysis of Exposure and Outcomes}

There were 138 sibling pairs in which one sibling was exposed to surgery/anesthesia and the other was not. In 11 of these sibling pairs both children had a subsequent behavioral/ developmental diagnosis, and in 107 sibling pairs neither child had a diagnosis. Of the remaining 20 discordant sibling pairs, in 9 sibling pairs, the exposed sibling had a subsequent diagnosis while the unexposed sibling did not, and in the other 11 sibling pairs, the unexposed sibling had a subsequent diagnosis while the exposed child did not. This resulted in a matched relative risk of $0.9(95 \%$ CI $0.6,1.4)$.

\section{Sensitivity Analyses}

To assess the potential magnitude of unmeasured confounding we examined the association of a developmental or behavioral diagnosis before the occurrence of a surgical procedure. Six hundred sixty-eight children underwent surgery when they were younger than 3 years accumulating a total of 1,483 person-years of follow-up from birth until time of surgery. Of these 668 children, 365 had a developmental or behavioral diagnosis before surgery occurred for an incidence rate of 24.6 diagnoses per 100 person years of observation (365 diagnoses / 1,483 person years $\times 100$ ).

Among 7,197 children who did not have surgery when they were younger than 3 years, 1,454 were diagnosed with a behavioral/developmental disorder before age 27 months (the average age at surgery for the 668 children) yielding an incidence rate of 18.6 diagnoses per 100 person years $(1,454$ diagnoses $/ 7,827$ person years $\times 100)$. The estimated incidence rate ratio between the two groups was 1.3 (95\% CI 1.2, 1.4).

\section{DISCUSSION}

In this nested cohort study the incidence of developmental and behavioral disorders in children exposed to anesthesia in the setting of surgery was 128.2 diagnoses per 1000 person-years compared to 56.3 diagnoses per 1000 person-years for the unexposed cohort. The adjusted estimated hazard ratio of 1.6 for any exposure is lower than that from our prior findings in a population from which this cohort was derived. ${ }^{13}$ By matching on sibling status, these results more precisely control for the effects of age, family and social environment and uterine exposures.

Our results are consistent with those of other investigators. As in a population-based retrospective study of cognitive outcomes, ${ }^{12}$ the effect of anesthesia and surgery could be seen to be dose-dependent. However, as in a twin-registry study, ${ }^{14}$ when restricted to pairwise concordant-discordant analysis, there was no effect, although dose-response was not measured in this study.

In this analysis, we attempted to further refine the important role that bias and confounding factors play in assessing any causal relationship between anesthesia and developmental or behavioral outcomes in children. Clinical studies ${ }^{27,} 28$ have demonstrated that children who 
require surgery at early ages differ in important ways from children who do not, and that those differences play a role in any potential risk attributed to anesthesia or surgery.

We, for example, excluded most of the ear-nose-throat cases for the reason that they very frequently involved procedures such as ear tube placement that would impact on the outcomes we were measuring. Although they might have been conducted for indications such as sleep apnea, we decided to keep in tonsillectomy and adenoidectomy. Our choice was guided by trying to balance those surgeries that were clearly and a priori potential confounders with the desire to be as inclusive as possible to increase the external validity of the study. For this reason, we excluded those ear-nose-throat cases which involved ear tube placement but not tonsillectomy and adenoidectomy, although they may have been conducted for indications such as sleep apnea.

Use of sibling controls is an effective way to minimize confounding by variables that are important yet difficult to measure, such as household and neighborhood environments. ${ }^{29} \mathrm{By}$ comparing the results of the present study to those of our previous analysis that did not use sibling controls, ${ }^{13}$ we can further describe and quantify the role of such confounding. Although the association between anesthesia in the setting of surgery and neurodevelopmental disorders remains statistically significant after controlling for sibships, socioeconomic status, age, home environment, parental factors, medical history, gender and (to a much less extent) genetic predisposition to neurodevelopmental disorders appear to account for nearly half of our previously observed effect size. ${ }^{13}$ Similarly, by demonstrating and measuring a significant association among a group of children for whom behavioral/ developmental diagnoses predated surgery/anesthesia, we conclude that an meaningful proportion of the association measured in the overall analysis of the sibling cohort may not be causally attributable to surgery/anesthesia.

We chose survival analytic methods controlling for clustering by sibling status, rather than conditional logistic regression or general estimating equations, so that we could more directly compare our results to our previous survival analyses. We supplemented that approach with a more restrictive pair-matched analysis. That our frequency-matched analysis did not closely approximate our pair-matched analysis can be attributed to two main reasons. First, the pair-matched analysis was based on a small subset of the study sample used in the frequency-matched analysis. There were insufficient numbers. An additional discordant sibling pair in either direction would have resulted in a meaningfully different result. Second, the pair-matched analysis presumably is far less susceptible to confounders than the unmatched analysis. Thus, the pair-matched analysis provides a more valid but less precise estimate than the frequency-matched analysis.

This study is subject to a number of limitations that require circumspection in interpreting these results. While the reliability of using Medicaid databases for analyzing behavioral and developmental diagnoses ${ }^{20}$ has been demonstrated, and the 635,758 births we identified represent $50.3 \%$ of all births in New York State for that time period, ${ }^{17}$ children born into Medicaid differ from the general population in ways that affect their health and medical care utilization $^{30}$ and that make them more likely to suffer mental illness. ${ }^{31}$

The rates of behavioral/developmental disorders in the population we studied were higher than those reported in the general population. ${ }^{32}$ In addition to reports of earlier and more frequent neurodevelopmental diagnoses in Medicaid children, ${ }^{33,34}$ multiple births are associated with increased risk of prematurity and low birth weight 35,36 and all that entails. Our exclusion criteria artificially minimized the denominators in the incidence calculations.

The administrative dataset did not contain any information on the zygosity of the sibling pairs. For this reason, and because dizygotic or fraternal twin births greatly outnumber 
monozygotic or identical twin births, the study can only be interpreted as a sibling cohort. The study cannot make observations about genetic differences or similarities as did the Bartels et al Dutch registry study. ${ }^{14}$ Although we do believe establishing sibling status helps to control for important variables, such as uterine environment, home atmosphere, parenting style and upbringing, educational systems and neighborhood or ecological characteristics, in which the units of analysis are populations or groups of people, rather than individuals. The dataset similarly did not contain information on which anesthetics were used and their duration of exposure.

While we, among others, have put large administrative health datasets to public health use, ${ }^{37,38}$ administrative data are a blunt instrument to assess possibly subtle neurocognitive outcomes. There will, inevitably, be measurement error, particularly in diagnoses which are abstracted by nonclinical personnel and must conform to insurance billing standards. Notably, such errors are likely to be "nondifferential" in epidemiological terms, because they are as likely among exposed as among unexposed and would be expected to bias results toward no or a null association. In this study, though, it is important to note that the large majority of outcomes were entered as "nonspecific delay." These assessments were likely to be just that, and many children were likely to recover and "catch up" from these delays. However, our dataset cannot capture those recoveries. There is no insurance billing code for that kind of return to a nondiagnosable status. This is likely to be the case for many administrative datasets.

Administrative data are also vulnerable to the issue of duplicate records. Medicaid, for example, may include records for the same diagnostic event on different dates if they are accepted as separate billable visits. This is a particular concern for outpatient visits for chronic conditions and may result in both an overestimation of absolute numbers of visits and relative measures of association. This problem may be compounded by beneficiaries who lose eligibility thus decreasing the denominator at risk. We attempted to limit outcomes to the first occurrence of a diagnosis during the period of Medicaid eligibility, and strictly normalized rates by person-years of observation within the Medicaid system.

While anesthesia may be independently and perhaps causally associated with behavioral and developmental pathology in young children, it will be difficult to establish causality based on the secondary analyses of observational epidemiological data. The population-level mixing of individual factors associated with both the indication for anesthesia and with neurodevelopmental diagnoses pose a formidable barrier to pursuing a counterfactual approach, where children differ solely on exposure to anesthesia. While we await the results of current and planned prospective clinical studies and trials, we can consider how the results of these and other observational epidemiological studies may inform additional research.

A productive line of inquiry may lie at the ecologic level. There is a large literature documenting the importance of social environment in educational attainment as well as in general morbidity and mortality. ${ }^{39-41}$ Surgery or anesthesia may interact with or lower the threshold for the causal effects of ecologic-level variables such as home or educational environment.

We suggest that while efforts directed toward prospective cohort studies be supported, additional analyses of existing data would most profitably contribute evidence to help guide future efforts if they compare and contrast different populations, examine the potential role of effect modifiers and mediators, more precisely measure and elucidate both exposures and outcomes or use analytic methods to address such issues as unmeasured confounding. 
Both clinicians and parents might rightly ask what, if any, changes or interventions in practice are warranted. At present, the strongest evidence is laboratory-based. While there is clinical evidence that anesthesia in the setting of surgery in children younger than three years is associated with subsequent developmental or behavioral diagnoses, the apparent association may well be due to confounding factors and a causal relationship remains unproven. This study similarly does not change current recommendations, and children who require surgery should not forgo any necessary procedure. Revision of current clinical guidelines must await more conclusive epidemiologic evidence from rigorously designed and meticulously executed studies.

\section{Acknowledgments}

Funding: This research was supported in part by grant UL1 RR024156 from the National Center for Research Resources and grant R01AA09963 from the National Institute on Alcohol Abuse and Alcoholism, National Institutes of Health. The funding agencies had no role in the design and conduct of the study; the collection, management, analysis, and interpretation of the data; or the preparation, review, or approval of the manuscript.

\section{Appendix}

List of ICD9 Codes Used to Identify Complications at Birth

\begin{tabular}{|l|l|}
\hline $\begin{array}{l}\text { Diagnostic } \\
\text { Codes }\end{array}$ & Diagnostic Category \\
\hline $001-139$ & ID parasites \\
\hline $140-239$ & Neoplasm \\
\hline $240-279$ & Endocrine, Metabolic, Immune \\
\hline $280-289$ & Hematologic \\
\hline $290-316$ & Mental including ETOH and Drug \\
\hline $317-319$ & Mental Retardation \\
\hline $320-326$ & CNS Inflammatory \\
\hline $330-337$ & CNS Degenerative \\
\hline $340-349$ & CNS Other \\
\hline $350-359$ & PNS \\
\hline $360-379$ & Eye and Adnexa \\
\hline $380-389$ & Ear and Mastoid \\
\hline $390-398$ & Rheumatic Disease \\
\hline $401-404$ & Hypertensive Disease \\
\hline $410-429$ & Cardiac \\
\hline $430-438$ & CVD \\
\hline $440-459$ & Circulatory including hemorrhage \\
\hline $460-519$ & Respiratory including pneumonia \\
\hline $520-549$ & GI \\
\hline $550-553$ & Hernia Abd Cavity \\
\hline $555-579$ & Other Intestinal including Liver \\
\hline $580-608$ & GU including Renal \\
\hline $610-629$ & Breast GYN \\
\hline $640-648$ & Pregnancy complications including hemorrhage \\
\hline
\end{tabular}




\begin{tabular}{|l|l|}
\hline $\begin{array}{l}\text { Diagnostic } \\
\text { Codes }\end{array}$ & Diagnostic Category \\
\hline $652-653$ & Malposition disproportion including hydrocephalus \\
\hline $654-655$ & Congenital malformation \\
\hline $656-659$ & Other Fetal Problems \\
\hline $660-669$ & Complications of Labor \\
\hline $670-675$ & Complications of puerperium \\
\hline $680-709$ & Dermatological \\
\hline $710-739$ & Musculoskeletal \\
\hline $740-742$ & Congenital Anomaly CNS \\
\hline $743-744$ & Congenital Anomaly EENT \\
\hline $745-747$ & Congenital Anomaly Cardiac \\
\hline $748-749$ & Congenital Anomaly Respiratory \\
\hline $750-759$ & Congenital Anomaly Other including Down s \\
\hline $760-767$ & Low Birth Weight (<2500 grams) \\
\hline 765 & Prematurity \\
\hline $768-770$ & Perinatal hypoxia \\
\hline $771-779$ & Perinatal infection hemorrhage hemeotological \\
\hline $780-799$ & Ill-defined signs symptoms \\
\hline V01-V02 & exposed to ID \\
\hline
\end{tabular}

\section{References}

1. Todd MM. Anesthetic neurotoxicity: the collision between laboratory neuroscience and clinical medicine. Anesthesiology. 2004; 101:272-273. [PubMed: 15277905]

2. Young C, Jevtovic-Todorovic V, Qin YQ, Tenkova T, Wang H, Labruyere J, Olney JW. Potential of ketamine and midazolam, individually or in combination, to induce apoptotic neurodegeneration in the infant mouse brain. Br J Pharmacol. 2005; 146:189-197. [PubMed: 15997239]

3. Olney JW, Young C, Wozniak DF, Jevtovic-Todorovic V, Ikonomidou C. Do pediatric drugs cause developing neurons to commit suicide? Trends Pharmacol Sci. 2004; 25:135-139. [PubMed: 15019268]

4. Olney JW, Wozniak DF, Farber NB, Jevtovic-Todorovic V, Bittigau P, Ikonomidou C. The enigma of fetal alcohol neurotoxicity. Ann Med. 2002; 34:109-119. [PubMed: 12108574]

5. Head BP, Patel HH, Niesman IR, Drummond JC, Roth DM, Patel PM. Inhibition of p75 neurotrophin receptor attenuates isoflurane-mediated neuronal apoptosis in the neonatal central nervous system. Anesthesiology. 2009; 110:813-825. [PubMed: 19293698]

6. Lunardi N, Ori C, Erisir A, Jevtovic-Todorovic V. General anesthesia causes long-lasting disturbances in the ultrastructural properties of developing synapses in young rats. Neurotox Res. 2010; 17:179-188. [PubMed: 19626389]

7. Stratmann G, May LD, Sall JW, Alvi RS, Bell JS, Ormerod BK, Rau V, Hilton JF, Dai R, Lee MT, Visrodia KH, Ku B, Zusmer EJ, Guggenheim J, Firouzian A. Effect of hypercarbia and isoflurane on brain cell death and neurocognitive dysfunction in 7-day-old rats. Anesthesiology. 2009; 110:849-861. [PubMed: 19293696]

8. Hayashi H, Dikkes P, Soriano SG. Repeated administration of ketamine may lead to neuronal degeneration in the developing rat brain. Paediatr Anaesth. 2002; 12:770-774. [PubMed: 12519135] 
9. Rovnaghi CR, Garg S, Hall RW, Bhutta AT, Anand KJ. Ketamine analgesia for inflammatory pain in neonatal rats: a factorial randomized trial examining long-term effects. Behav Brain Funct. 2008; 4:35. [PubMed: 18687139]

10. Davidson AJ, McCann ME, Morton NS, Myles PS. Anesthesia and outcome after neonatal surgery: the role for randomized trials. Anesthesiology. 2008; 109:941-944. [PubMed: 19034085]

11. Sun LS, Li G, Dimaggio C, Byrne M, Rauh V, Brooks-Gunn J, Kakavouli A, Wood A. Anesthesia and neurodevelopment in children: time for an answer? Anesthesiology. 2008; 109:757-761. [PubMed: 18946281]

12. Wilder RT, Flick RP, Sprung J, Katusic SK, Barbaresi WJ, Mickelson C, Gleich SJ, Schroeder DR, Weaver AL, Warner DO. Early exposure to anesthesia and learning disabilities in a populationbased birth cohort. Anesthesiology. 2009; 110:796-804. [PubMed: 19293700]

13. DiMaggio C, Sun LS, Kakavouli A, Byrne MW, Li G. A retrospective cohort study of the association of anesthesia and hernia repair surgery with behavioral and developmental disorders in young children. J Neurosurg Anesthesiol. 2009; 21:286-291. [PubMed: 19955889]

14. Bartels M, Althoff RR, Boomsma DI. Anesthesia and cognitive performance in children: no evidence for a causal relationship. Twin Res Hum Genet. 2009; 12:246-253. [PubMed: 19456216]

15. Centers for Medicare and Medicaid Services. Medicaid Analytic eXtract (MAX) General Information. 2006. 2006

16. Foundation KF. Kaiser Commission on Medicaid and the Uninsured. 2008. 2008

17. New York State Department of Health. Vital Statistics of New York State. 2009. 2009

18. AMA. International classification of diseases, 9th revision, clinical modification : physician ICD-9-CM, 2005. AMA Press; Chicago, Ill: 2004.

19. Ala A, Stanca CM, Bu-Ghanim M, Ahmado I, Branch AD, Schiano TD, Odin JA, Bach N. Increased prevalence of primary biliary cirrhosis near Superfund toxic waste sites. Hepatology. 2006; 43:525-531. [PubMed: 16496326]

20. Walkup JT, Boyer CA, Kellermann SL. Reliability of Medicaid claims files for use in psychiatric diagnoses and service delivery. Adm Policy Ment Health. 2000; 27:129-139. [PubMed: 10826215]

21. Kelly PJ. A Review of Software Packages for Analyzing Correlated Survival Data. the American Statistician. 2004; 58:337-442.

22. Braver ER, Scerbo M, Kufera JA, Alexander MT, Volpini K, Lloyd JP. Deaths among drivers and right-front passengers in frontal collisions: redesigned air bags relative to first-generation air bags. Traffic Inj Prev. 2008; 9:48-58. [PubMed: 18338295]

23. Cummings P, Wells JD, Rivara FP. Estimating seat belt effectiveness using matched-pair cohort methods. Accid Anal Prev. 2003; 35:143-149. [PubMed: 12479905]

24. Greenland, S. Chapter 16: Applications of stratified analysis methods. In: Rothman, SGKJ., editor. Modern Epidemiology. Lippincott Williams \& Wilkins; Philadelphia: 1998.

25. SAS. SAS 9.1.3. 2006.

26. R Development Core Team. R: A Language and Environment for Statistical Computing. 2008. 2008

27. Kalkman CJ, Peelen L, Moons KG, Veenhuizen M, Bruens M, Sinnema G, de Jong TP. Behavior and development in children and age at the time of first anesthetic exposure. Anesthesiology. 2009; 110:805-812. [PubMed: 19293699]

28. Sprung J, Flick RP, Wilder RT, Katusic SK, Pike TL, Dingli M, Gleich SJ, Schroeder DR, Barbaresi WJ, Hanson AC, Warner DO. Anesthesia for cesarean delivery and learning disabilities in a population-based birth cohort. Anesthesiology. 2009; 111:302-310. [PubMed: 19602960]

29. Susser E, Eide MG, Begg M. Invited commentary: The use of sibship studies to detect familial confounding. Am J Epidemiol. 2010; 172:537-539. [PubMed: 20631043]

30. Salsberry PJ, Chipps E, Kennedy C. Use of general medical services among Medicaid patients with severe and persistent mental illness. Psychiatr Serv. 2005; 56:458-462. [PubMed: 15812097]

31. Satcher DS. Executive summary: a report of the Surgeon General on mental health. Public Health Rep. 2000; 115:89-101. [PubMed: 10968589] 
32. Blanchard LT, Gurka MJ, Blackman JA. Emotional, developmental, and behavioral health of American children and their families: a report from the 2003 National Survey of Children's Health. Pediatrics. 2006; 117:e1202-12. [PubMed: 16740820]

33. Mandell DS, Listerud J, Levy SE, Pinto-Martin JA. Race differences in the age at diagnosis among medicaid-eligible children with autism. Journal of the American Academy of Child and Adolescent Psychiatry. 2002; 41:1447-1453. [PubMed: 12447031]

34. Mandell DS, Novak MM, Zubritsky CD. Factors associated with age of diagnosis among children with autism spectrum disorders. Pediatrics. 2005; 116:1480-6. \%U http://www.ncbi.nlm.nih.gov/ pubmed/16322174. [PubMed: 16322174]

35. Ghai V, Vidyasagar D. Morbidity and mortality factors in twins. An epidemiologic approach. Clin Perinatol. 1988; 15:123-140. [PubMed: 3286088]

36. Kiely JL. The epidemiology of perinatal mortality in multiple births. Bull N Y Acad Med. 1990; 66:618-637. [PubMed: 2282443]

37. DiMaggio C, Galea S, P. M. Population psychiatric medication subscription rates following a terrorist attack. Prehospital Disast Med. 2007; 22:479-484.

38. DiMaggio C, Durkin M. Child pedestrian injury in an urban setting: descriptive epidemiology. Acad Emerg Med. 2002; 9:54-62. [PubMed: 11772671]

39. Backlund E, Sorlie PD, Johnson NJ. A comparison of the relationships of education and income with mortality: the National Longitudinal Mortality Study. Soc Sci Med. 1999; 49:1373-1384. [PubMed: 10509827]

40. Batty GD, Shipley MJ, Mortensen LH, Boyle SH, Barefoot J, Gronbaek M, Gale CR, Deary IJ. IQ in late adolescence/early adulthood, risk factors in middle age and later all-cause mortality in men: the Vietnam Experience Study. J Epidemiol Community Health. 2008; 62:522-531. [PubMed: 18477751]

41. Ford ES, Loucks EB, Berkman LF. Social integration and concentrations of C-reactive protein among US adults. Ann Epidemiol. 2006; 16:78-84. [PubMed: 16271297] 


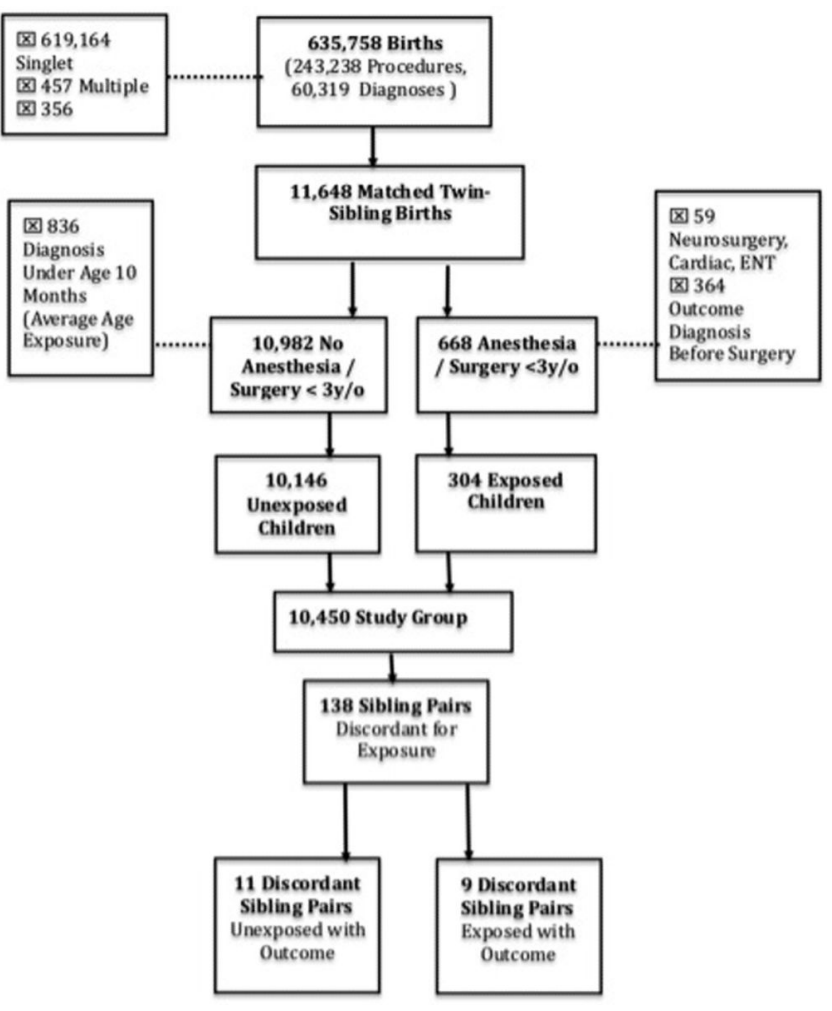

Figure 1.

Selection of the Study Subjects. New York Medicaid 1999-2005. 


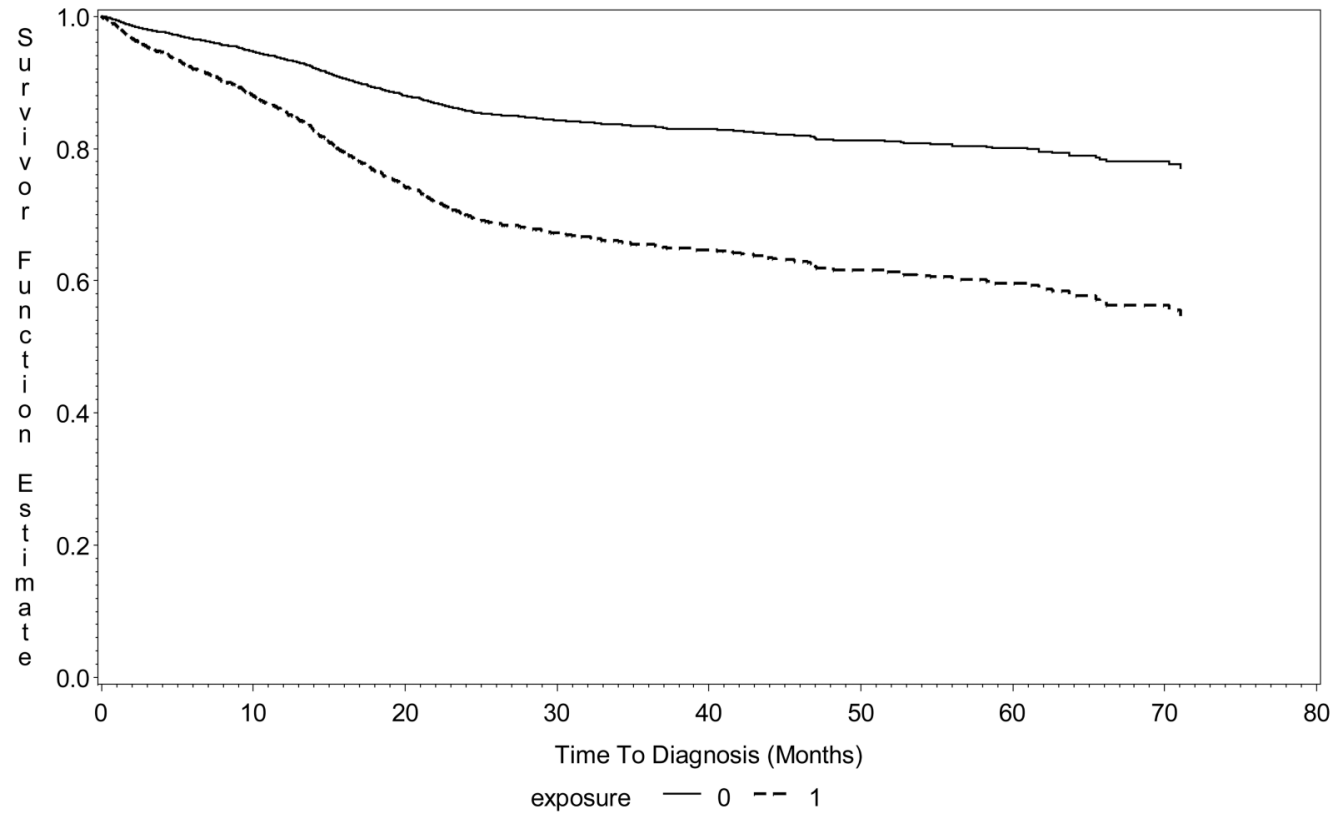

Figure 2.

Kaplan-Meier Survival Curve time (in months) for individual twins with any exposure to surgery/anesthesia (dashed line) to twins not exposed to surgery/anesthesia (solid line) for subsequent behavioral/developmental outcomes, adjusted for birth-related complications and gender. Log-rank test chi square=7.2 ( $\mathrm{p}=0.008)$. Analysis based on 304 exposed and 10,146 unexposed individual twins, New York State Medicaid data 1999-2005. 
Table 1

Frequency and percentage of surgical procedures, based on ICD-9 procedure codes, siblings born into New York City Medicaid 1999-2005.

\begin{tabular}{|c|c|c|}
\hline Procedure & $\begin{array}{l}\text { All Surgery } \\
\text { Anesthesia } \\
\text { Exposures } \\
(\%)\end{array}$ & $\begin{array}{l}\text { Exposed to } \\
\text { Surgery / } \\
\text { Anesthesia } \\
\text { Without Prior } \\
\text { Behavioral } \\
\text { Diagnosis (\%) }\end{array}$ \\
\hline Inguinal Hernia Repair & $364(54.5 \%)$ & $199(65.3 \%)$ \\
\hline Repair Of Gastroschisis & $162(24.2 \%)$ & $13(4.3 \%)$ \\
\hline Exploratory Laparotomy & $34(5.1 \%)$ & $5(1.7 \%)$ \\
\hline Appendectomy & $22(3.3 \%)$ & $19(6.3 \%)$ \\
\hline Pyloromyotomy & $16(2.4 \%)$ & $16(5.3 \%)$ \\
\hline Tonsillectomy/Adenoidectomy & $14(2.1 \%)$ & $7(2.3 \%)$ \\
\hline Partial Small Bowel Resection & $13(1.9 \%)$ & $13(4.3 \%)$ \\
\hline Hemicolectomy & $7(1.0 \%)$ & $7(2.3 \%)$ \\
\hline Create Esophagastric Sphincter & $6(0.9 \%)$ & $20.7 \%)$ \\
\hline Closed Fracture Reduction Femur & $6(0.9 \%)$ & $6(2.0 \%)$ \\
\hline Closed Reduction Internal Fixation Humerus & $5(0.7 \%)$ & $3(1.0 \%)$ \\
\hline Left Hemicolectomy & $3(0.4 \%)$ & $3(1.0 \%)$ \\
\hline Small Bowel Stoma Closure & $3(0.4 \%)$ & $1(0.3 \%)$ \\
\hline Cleft Palate Correction & $2(0.3 \%)$ & $2(0.7 \%)$ \\
\hline Incision Perianal Abscess & $2(0.3 \%)$ & $2(0.7 \%)$ \\
\hline Laparoscopic Procedure & $2(0.3 \%)$ & $1(0.3 \%)$ \\
\hline Clubfoot Release & $2(0.3 \%)$ & $2(0.7 \%)$ \\
\hline Gastrostomy Closure & $1(0.1 \%)$ & 0 \\
\hline Closed Large Bowel Biopsy & $1(0.1 \%)$ & 0 \\
\hline Sigmoidectomy & $1(0.1 \%)$ & $1(0.3 \%)$ \\
\hline Ileostomy & $1(0.1 \%)$ & $1(0.3 \%)$ \\
\hline Large Bowel Stoma Closure & $1(0.1 \%)$ & $1(0.3 \%)$ \\
\hline Total & $668(100 \%)$ & $304(100 \%)$ \\
\hline
\end{tabular}


Table 2

Frequency and proportion of behavioral/developmental diagnoses among 304 children exposed to surgery/ anesthesia with no history of behavioral/developmental diagnoses vs. 10,982 unexposed children followed from age 10 months. New York State Medicaid Analytic Extract files, 1999-2005.

\begin{tabular}{|l|r|r|}
\hline Diagnosis & Exposed & Unexposed \\
\hline Development Delay & $62(82.7 \%)$ & $692(77.2 \%)$ \\
\hline Language Disorder & $5(6.7 \%)$ & $127(14.2 \%)$ \\
\hline Attention Deficit & $4(5.3 \%)$ & $30(3.3 \%)$ \\
\hline Coordination Disorder & $1(1.3 \%)$ & $14(1.6 \%)$ \\
\hline Conduct Disorder & $1(1.3 \%)$ & $20(2.2 \%)$ \\
\hline Other* & $2(2.7 \%)$ & $13(1.5 \%)$ \\
\hline Total & $75(100 \%)$ & $896(100 \%)$ \\
\hline
\end{tabular}


Table 3

Hazard ratios and $95 \%$ Confidence Intervals of behavioral/developmental outcomes in children exposed to surgery/anesthesia before age 3, adjusted for clustering by sibling status. New York State Medicaid Data 1999-2005.

\begin{tabular}{|l|l|l|}
\hline $\begin{array}{l}\text { Number of Surgeries Under } \\
\text { 3 Years of Age }\end{array}$ & Hazard Ratio & 95\% CI \\
\hline 0 & 1 & \\
\hline 1 & 1.1 & $(0.8,1.4)$ \\
\hline 2 & 2.8 & $(2.5,3.1)$ \\
\hline 3 or More & 4.0 & $(3.5,4.5)$ \\
\hline $\begin{array}{l}\text { History of Complication at } \\
\text { Birth }\end{array}$ & & \\
\hline No & 1 & \\
\hline Yes & 1.5 & $(1.3,1.6)$ \\
\hline Gender & & \\
\hline Female & 1 & $(1.3,1.5)$ \\
\hline Male & 1.4 & \\
\hline
\end{tabular}

